DOI: 10.12957/demetra.2016.23369

\title{
Acesso aos alimentos e segurança alimentar e nutricional: avaliação da situação em municípios baianos
}

\author{
Access to food and food and nutrition security: situation assessment in municipalities \\ in Bahia, Brazil
}

Laís Santos Calmon

Sandra Maria Chaves dos Santos'

Marlus Henrique Queiróz Pereira²

1 Universidade Federal da Bahia, Escola de Nutrição, Departamento das Ciências da Nutrição. Salvador-BA, Brasil.

2 Universidade Federal do Oeste da Bahia, Centro das Ciências Biológicas e da Saúde. Barreiras-BA, Brasil.

Financiamento: 0 trabalho foi subvencionado pela Fundação de Amparo à Pesquisa do Estado da Bahia - Fapesb, 19.573.128.2586.

Correspondência / Correspondence Laís Santos Calmon

E-mail: laiscalmon@hotmail.com

\section{Resumo}

Objetivo: Avaliar a situação de Segurança Alimentar e Nutricional nos municípios do Estado da Bahia e seus principais determinantes na dimensão de Acesso aos Alimentos. Métodos: Aplicou-se um protocolo de indicadores que avalia a Segurança Alimentar e Nutricional em âmbito municipal, englobando quatro dimensões (disponibilidade, acesso e consumo de alimentos e utilização biológica de nutrientes). Foram utilizados dados secundários dos municípios baianos, disponíveis em bases de dados on-line de acesso público. Foram calculadas medidas de prevalência e verificada a associação entre as variáveis do estudo. Resultados: Nos municípios avaliados, observou-se que 119 (28,7\%) estavam em Insegurança Alimentar leve e 286 (69,1\%), em Insegurança Alimentar moderada. Na dimensão Acesso aos Alimentos houve predominância de Insegurança Alimentar moderada (71,5\%), e os indicadores que mais contribuíram para esse quadro foram proporção de pessoas com baixa renda e percentual da população com 15 anos ou mais e menos de 4 anos de estudo. Houve associação significativa entre a situação de Insegurança Alimentar nos municípios, considerando todas as dimensões, e o estado de Insegurança Alimentar na dimensão de Acesso aos Alimentos $(\mathrm{p}<0,000)$. E entre a situação de Insegurança Alimentar na dimensão de Acesso aos Alimentos e menores Índices de Desenvolvimento Humano Municipal $(p<0,000)$. Conclusão: Apesar da dimensão de Acesso aos Alimentos ter forte relação com Insegurança Alimentar, não se mostrou suficiente para explicá-la isoladamente nos municípios baianos. A aplicação do protocolo adotado pode auxiliar gestores a definir ações estratégicas para enfrentamento do problema. 
Palavras-chave: Segurança Alimentar e Nutricional. Acesso aos Alimentos. Municípios baianos. Indicadores.

\section{Abstract}

Objective: To evaluate the situation of the food and nutrition security at the municipalities of Bahia's State and the main determinants in the dimension of access to feeding. Methods: We applied a protocol of indicators that evaluates food and nutrition security covering four dimensions (availability, food access and consumption and biological use of nutrients). Secondary data from the Bahia's municipalities (available in public online databases) were used. Prevalence measures were calculated and associations were verified between the variables of the study. Results: In the municipalities evaluated, it was noted that 119 (28.7\%) were in light Food Insecurity and 286 (69.1\%) were in moderate Food Insecurity. For the Access to feeding dimension, there was a predominance of moderate Food Insecurity (71.5\%) and the indicators that more contributed to this framework were the proportion of people with low income and percentage of population with 15 years or older and less than 4 years of schooling. There was a meaningful association between Food Insecurity in the municipalities, considering all the dimensions and the status of Food Insecurity at the dimension of Access to Food $(\mathrm{p}<0.000)$, and between the situation of Food Insecurity at the dimension of Access to Food and lowest Municipal Human Development Index $(\mathrm{p}<0.000)$. Conclusion: Although of the dimension Access to Food has strongly related to Food Insecurity, it did not show enough to explain it individually in Bahia's municipalities. The application of the protocol adopted can help managers to define strategical actions to face the problem.

Key words: Food and Nutrition Security. Acess to Food. Bahia's Counties. Indicators.

\section{Introdução}

Nas últimas décadas, o debate sobre Segurança Alimentar e Nutricional (SAN) no Brasil tem se tornado mais relevante, sendo discutido pelo Estado e setores da sociedade civil organizada., ${ }^{1,2}$

A SAN no Brasil é definida pela Lei Orgânica de Segurança Alimentar e Nutricional (Losan) como

a garantia da realização do direito de todos ao acesso regular e permanente a alimentos de qualidade, em quantidade suficiente, sem comprometer o acesso a outras necessidades essenciais, tendo como 
base práticas alimentares promotoras de saúde que respeitem a diversidade cultural e que sejam ambiental, cultural, econômica e socialmente sustentáveis. ${ }^{3}$

Esse conceito é fruto de diversas conquistas em relação à garantia de cidadania e direito humano à alimentação e destina-se a aproximar as quatro dimensões de SAN, que são: disponibilidade de alimentos (DA), acesso aos alimentos (AA), consumo de alimentos (CA) e utilização biológica de nutrientes (UBN), considerando também suas inter-relações. ${ }^{4,5}$

Nesse contexto, a SAN tem sido foco no desenvolvimento de ações e políticas públicas no Brasil que visam uma lógica integrada de atuação e abrangem diversas áreas do governo, como a saúde, a produção agrícola, o abastecimento alimentar, a geração de emprego e renda, a educação alimentar, a segurança e qualidade dos alimentos, assim como as ações diretas de ampliação do acesso aos alimentos, como a transferência direta de renda., ${ }^{4,6}$

Estudos sobre a prevalência de Segurança Alimentar e Insegurança Alimentar (IA) têm sido realizados no Brasil. Segundo dados da última Pesquisa Nacional de Amostragem por Domicílio (PNAD), realizada pelo Instituto Brasileiro de Geografia e Estatística (IBGE) em 2013, no Brasil, adotando a Escala Brasileira de Insegurança Alimentar (EBIA), ocorreu aumento na situação de domicílios particulares em SAN para 77,4\%, sendo registrado anteriormente 65,1 \% e 69,8 \%, em 2004 e 2009, respectivamente. Porém, a IA, em algum grau, ainda atinge cerca de 14,7 milhões de domicílios brasileiros (22,6\%), e as situações mais preocupantes são observadas nas regiões Norte e Nordeste do país (36,1\% e 38,1\%, respectivamente). Na Bahia, a prevalência de IA atinge 37,8\% dos domicílios, ou seja, este problema ainda afeta uma grande parcela da população brasileira e baiana. ${ }^{7}$

Um fator decisivo para esta situação de IA que persiste no Brasil é a dificuldade ao acesso regular e permanente aos alimentos. ${ }^{8}$ Seu acesso físico e econômico concretiza-se quando todos os cidadãos têm a possibilidade de obtê-los de forma idônea, por meio da produção, compra, caça, ou troca. Essa dimensão de SAN é extremamente complexa e engloba a disponibilidade de recursos para aquisição de alimentos pelas famílias e de todas as necessidades básicas necessárias para a garantia de SAN, além dos fatores que afetam os recursos disponíveis. ${ }^{9}$

As avaliações nacionais de SAN realizadas pelo IBGE com uso da ferramenta da EBIA buscam estimar as prevalências da situação de segurança alimentar. ${ }^{7}$ No entanto, a metodologia utilizada não contempla todas as dimensões da avaliação da SAN, e os dados produzidos não alcançam o nível municipal, lócus de implementação de diversas políticas públicas de enfrentamento de IA. Nessa perspectiva, o Núcleo de Nutrição e Políticas Públicas (NNPP) da Universidade Federal da Bahia (UFBA) vem incrementando estudos voltados a desenvolver e aplicar metodologia para avaliação da SAN e IA em nível municipal, englobando as quatro dimensões, como descrito por Panelli-Martins, Santos \& Assis e Pereira, sendo mais uma opção para avaliar um fenômeno tão complexo., ${ }^{1,5}$ 
Todas as dimensões acima referidas são relevantes para determinar a situação de SAN de uma população ou de um município. No entanto, estudos têm destacado a relevância da dimensão de acesso aos alimentos, na qual se considera fatores como renda mensal essencial para aquisição de alimentos. Assim, conhecer a prevalência de IA e SAN em âmbito municipal e aprofundar os estudos nos determinantes da dimensão de acesso dos alimentos pode apoiar o estabelecimento de objetivos prioritários no planejamento e desenvolvimento das políticas públicas em nível municipal, além do monitoramento da efetividade destas ações, o que pode contribuir para assegurar condições favoráveis para o alcance da SAN, principalmente em municípios mais vulneráveis. ${ }^{10}$

Dessa forma, o presente estudo avaliou, a partir de uma metodologia que adota um protocolo de indicadores, a situação de SAN nos municípios do Estado da Bahia e seus principais determinantes na dimensão de acesso aos alimentos.

\section{Metodologia}

O presente estudo integra um projeto maior titulado "Rede Baiana para Desenvolvimento e Aplicação de Tecnologias Sociais de Avaliação da Segurança Alimentar e Nutricional - REDETECSAN/BA", o qual é desenvolvido por pesquisadores do Núcleo de Nutrição e Políticas Públicas (NNPP) da Escola de Nutrição da Universidade Federal da Bahia (ENUFBA) e financiado pela Fundação de Amparo à Pesquisa do Estado da Bahia (Fapesb).

\section{Cenário de estudo}

O estudo foi proposto para os municípios localizados no Estado da Bahia (417 municípios), no entanto, não foi possível encontrar todos os dados secundários disponíveis necessários para a aplicação do protocolo de indicadores em todos os municípios baianos.

\section{Instrumento de análise}

A avaliação da situação de SAN nos municípios foi realizada mediante a aplicação de um protocolo de indicadores proposto por Panelli-Martins, e atualizado por Pereira. ${ }^{1,11}$ Este protocolo de indicadores tem o intuito de avaliar a SAN em toda a sua magnitude, sendo uma ferramenta de baixo custo, boa operacionalização e eficaz na análise de SAN e IA em âmbito municipal. O protocolo, originalmente, foi composto por 25 indicadores, agrupados em quatro dimensões de análise da SAN, distribuído da seguinte forma: ${ }^{1}$

- Disponibilidade de Alimentos (DA), 5 indicadores, que estão relacionados ao transporte, à produção e à comercialização de alimentos; 
- Acesso aos alimentos (AA), 11 indicadores, englobando fatores sociais, econômicos e culturais que interferem na aquisição dos alimentos;

- Consumo de alimentos (CA), 5 indicadores, relacionados ao estado de saúde e nutrição que refletem no padrão nutricional;

- Utilização biológica de nutrientes (UBN), 4 indicadores, os quais se relacionam às condições de acesso a serviços sociais, de saneamento e de saúde e suas repercussões na alimentação e nutrição do indivíduo/população.

Foram excluídos deste estudo dois indicadores da dimensão CA, a saber: taxa de prevalência de déficit de estatura para a idade em crianças < 5 anos e excesso de peso em mulheres, devido à indisponibilidade de informações nos bancos de dados de acesso público. Dessa forma, foram calculados 23 indicadores do protocolo original.

Segundo os autores citados, os indicadores que passaram a integrar o protocolo transpassam diversos aspectos da sociedade, como saúde, estado nutricional, educação, renda, emprego, clima, saneamento, água, acesso à terra, agricultura, entre outros. Estes indicadores foram os que melhor se adaptaram às propriedades como relevância social, confiabilidade e a validade de constructo, e características desejáveis como sensibilidade, especificidade, inteligibilidade, periodicidade, factibilidade e historicidade..$^{1,12}$

Após uma visão geral da situação de SAN nos municípios, faz-se neste estudo uma análise mais aprofundada sobre os resultados obtidos na dimensão de acesso aos alimentos, seus fatores determinantes e a relação destes com o Índice de Desenvolvimento Humano Municipal (IDHM).

\section{Coleta de dados}

Os indicadores foram calculados a partir de dados secundários, referentes ao período de 2010-2014, obtidos em bases de acesso público on-line: Departamento de Informática do Sistema Único de Saúde (DATASUS), Instituto Brasileiro de Geografia e Estatística (IBGE), Sistema de Informação da Atenção Básica (SIAB), assim como nos relatórios sociais da Secretaria de Avaliação e Gestão da Informação (SAGI) do Ministério de Desenvolvimento Social e Combate a Fome (MDS). Todos os dados coletados foram armazenados em uma planilha do protocolo, utilizando o software Microsoft Office Excel versão 2007.

\section{Análise e processamento de dados}

O parâmetro de classificação usado e a pontuação dos indicadores foram realizados conforme a metodologia do protocolo desenvolvido por Pereira, sendo atribuído a cada indicador uma 
pontuação de 0 a $10 .{ }^{1} \mathrm{O}$ valor 0 é atribuído quando o resultado é considerado inadequado, indicativo de risco de IA; o valor 5 para resultados aceitáveis e o valor 10, associado com resultados adequados para garantia da SAN. Alguns indicadores têm resultados dicotômicos, ou seja, o indicador pontua o mínimo para a resposta negativa (0 ponto) e o máximo em caso de resposta positiva (10 pontos). Dessa forma, a pontuação dos indicadores foi distribuída por dimensão: DA, 0 a 50 pontos; AA, 0 a 110 pontos; CA, 0 a 30 pontos; e por fim, UBN, 0 a 40 pontos. Para fazer equivaler a importância entre as dimensões, as quais contam com número diferenciado de indicadores, portanto somam pontuações diversas, foi realizada a frequência relativa por dimensão e a média ponderada das mesmas. Isto se fez tendo por base o entendimento de que cada uma das quatro dimensões detém igual força na construção da SAN. Assim, quanto maior a pontuação em cada dimensão, melhores as condições de promoção da SAN.

Utilizando esse sistema de pontos, foi adotada a escala de classificação da SAN/IA, relativa ao percentual de desempenho por quartis, na qual cada intervalo corresponde a diferentes gradientes de SAN ou IA (Quadro 1).

Quadro 1. Escala de pontuação (percentuais) e categorias para avaliação da SAN municipal com uso de um protocolo de indicadores. Salvador - BA, 2015.

\begin{tabular}{|c|c|c|c|}
\hline \multicolumn{4}{|c|}{ ESCALA DE CLASSIFICAÇÃO DA SAN } \\
\hline IA GRAVE & IA MODERADA & IA LEVE & SAN \\
\hline 0 a $24,99 \%$ & 25 а $49,99 \%$ & 50 a $74,99 \%$ & 75 a $100 \%$ \\
\hline $\begin{array}{c}\text { Resultados dos } \\
\text { indicadores } \\
\text { sinalizam para } \\
\text { existência de } \\
\text { muitos fatores } \\
\text { comprometedores } \\
\text { da SAN, implicando } \\
\text { exposição à } \\
\text { privação de } \\
\text { alimentos e fome. }\end{array}$ & $\begin{array}{l}\text { Resultados dos } \\
\text { indicadores } \\
\text { sinalizam para } \\
\text { existência de } \\
\text { alguns fatores } \\
\text { comprometedores } \\
\text { da SAN, com } \\
\text { risco de situações } \\
\text { de privação de } \\
\text { alimentos e fome. }\end{array}$ & $\begin{array}{l}\text { Resultados dos } \\
\text { indicadores } \\
\text { sinalizam para } \\
\text { existência de } \\
\text { alguns fatores } \\
\text { comprometedores } \\
\text { da SAN, sem } \\
\text { caracterizar risco } \\
\text { de privação de } \\
\text { alimentos e fome. }\end{array}$ & $\begin{array}{c}\text { Resultados dos } \\
\text { indicadores } \\
\text { sinalizam para } \\
\text { condições favoráveis } \\
\text { à promoção da SAN } \\
\text { no município. }\end{array}$ \\
\hline
\end{tabular}

Fonte: Pereira. ${ }^{1}$ 
O IDHM é um indicador que segue as mesmas três dimensões (saúde, educação e renda) do Índice de Desenvolvimento Humano (IDH) global, mas adaptando a metodologia global ao contexto brasileiro e aos indicadores nacionais disponíveis. É composto por três componentes: IDHM Longevidade, IDHM Educação e IDHM Renda. E mensurado por uma escala numérica que varia entre 0 e 1 , em que valores mais próximos de 1 demonstram maior desenvolvimento humano de um município. ${ }^{13}$ Para classificação do IDHM, empregou-se o parâmetro publicado pelo Programa das Nações Unidas para o Desenvolvimento (PNUD), em que as Faixas de Desenvolvimento Humano Municipal são muito baixo (0 a 0,499), baixo (0,500 a 0,599), médio (0,600 a 0,699), alto $(0,700$ a $0,799)$ e muito alto $(0,800$ a 1$) \cdot{ }^{13}$

Com o software Statistical Packadge Social of Sciences (SPSS) para Windows (versão 17.0) foi feita análise descritiva para medir a frequência das variáveis do estudo. Em seguida, para constatar a associação entre a dimensão AA e a IA nos municípios do Estado da Bahia e a associação entre a situação de IA na dimensão de Acesso aos Alimentos e o Índice de Desenvolvimento Humano Municipal, foi aplicado o teste qui-quadrado, considerando significante a associação com p-valor $\leq 0,05$. Além disso, para realizar a associação, foram agrupadas as variáveis IA moderada/IA grave e as faixas de Desenvolvimento Humano Municipal Muito baixo/Baixo e Alto/Muito alto.

\section{Resultados}

Análise geral da situação de Segurança Alimentar e Nutricional nos municípios

Nos municípios avaliados, observou-se que dois estavam em situação de SAN, 119 (28,7 \%), em IA leve, 286 (69,1\%), em IA moderada e 7 (1,7\%), em IA grave (Tabela 1).

Tabela 1. Prevalência de SAN e IA em municípios do Estado da Bahia. Salvador - BA, 2015.

\begin{tabular}{lcc}
\hline \multicolumn{1}{c}{ Variável } & $\mathrm{n}$ & $\%$ \\
\hline Segurança Alimentar e Nutricional & 2 & 0,5 \\
Insegurança Alimentar leve & 119 & 28,7 \\
Insegurança Alimentar moderada & 286 & 69,1 \\
Insegurança Alimentar grave & 7 & 1,7 \\
\hline Total & 414 & 100,0 \\
\hline
\end{tabular}


A classificação da situação de SAN por dimensão e a sua influência no resultado global podem ser observadas nos resultados mostrados na tabela 2.

Tabela 2. Prevalência de SAN e IA em âmbito municipal, segundo as dimensões de avaliação da SAN. Salvador - BA, 2015.

\begin{tabular}{ccccc}
\hline \multirow{2}{*}{ Variável } & Disponibilidade & Acesso & Consumo & Utilização \\
& $\mathrm{n}(\%)$ & $\mathrm{n}(\%)$ & $\mathrm{n}(\%)$ & $\mathrm{n}(\%)$ \\
\cline { 2 - 4 } SAN & $38(9,2)$ & $0(0,0)$ & $38(9,2)$ & $54(13,0)$ \\
IA leve & $150(36,2)$ & $60(14,5)$ & $222(53,6)$ & $188(45,4)$ \\
IA moderada & $140(33,8)$ & $296(71,5)$ & $88(21,3)$ & $153(37,0)$ \\
IA grave & $86(20,8)$ & $58(14,0)$ & $66(15,9)$ & $19(4,6)$ \\
Total & $414(100,0)$ & $414(100,0)$ & $414(100,0)$ & $414(100,0)$ \\
\hline
\end{tabular}

Na dimensão DA, a maioria dos municípios estava em IA leve (36,2\%) e 33,8\% em IA moderada. Esta foi a dimensão que apresentou a maior prevalência de IA grave (20,8\%).

Por outro lado, as dimensões CA e UBN apresentaram as maiores prevalências de SAN (9,2\% e $13 \%$ respectivamente) e a forma mais leve de IA (53,6\% e 45,4\%, respectivamente).

\section{Análise da Segurança Alimentar e Nutricional com foco na Dimensão de Acesso aos Alimentos}

Na dimensão AA houve predominância de IA moderada (71,5\%). A IA leve (14,5\%) e a IA grave (14\%) se apresentaram com percentuais muito próximos. Nenhum município nesta dimensão foi classificado com condições favoráveis para garantia de SAN. Esses resultados podem ser explicados pelos percentuais obtidos nos indicadores da dimensão de acesso descritos na tabela 3 . 
Tabela 3. Análise dos indicadores da dimensão de Acesso aos Alimentos em municípios da Bahia. Salvador - BA, 2015.

\begin{tabular}{|c|c|c|c|}
\hline Indicador/dimensão & $\begin{array}{c}\text { Adequado } \\
\%\end{array}$ & $\begin{array}{c}\text { Aceitável } \\
\%\end{array}$ & $\begin{array}{c}\text { Inadequado } \\
\%\end{array}$ \\
\hline \multicolumn{4}{|l|}{ Acesso aos Alimentos } \\
\hline Média da renda domiciliar mensal per capita & 58,0 & - & 42,0 \\
\hline Índice de Gini da renda domiciliar & 24,2 & 75,4 & 0,5 \\
\hline Razão de renda & 72,0 & 17,4 & 12,8 \\
\hline $\begin{array}{l}\% \text { de pessoas com renda domiciliar mensal } \\
\text { per capital< }<1 / 2 \mathrm{SM}^{*}\end{array}$ & 0,7 & 9,4 & 89,9 \\
\hline $\begin{array}{l}\% \text { de desemprego na população } \\
\text { economicamente ativa de } 16 \text { anos e mais }\end{array}$ & 17,6 & 78,7 & 3,6 \\
\hline $\begin{array}{l}\% \text { de famílias de baixa renda atendidas } \\
\text { pelo } \mathrm{PBF}^{* *}\end{array}$ & 46,9 & 43,5 & 9,7 \\
\hline $\begin{array}{l}\text { Taxa de analfabetismo de pessoas com } 15 \\
\text { anos ou mais }\end{array}$ & 2,7 & 37,9 & 59,4 \\
\hline $\begin{array}{l}\text { \% da população com } 15 \text { anos ou mais e } \\
\text { menos de } 4 \text { anos de estudo }\end{array}$ & 3,4 & 7,0 & 89,6 \\
\hline $\begin{array}{l}\mathrm{N}^{\circ} \text { de moradores por domicílios } \\
\text { particulares }\end{array}$ & 22,5 & - & 77,5 \\
\hline$\%$ de famílias chefiadas por mulheres & 5,8 & 9,4 & 84,8 \\
\hline Existência de equipamentos públicos de SAN & 2,7 & 8,7 & 88,6 \\
\hline
\end{tabular}

*Salário Mínimo. ** Programa Bolsa Família.

Os indicadores proporção de pessoas com baixa renda e percentual da população com 15 anos ou mais e menos de 4 anos de estudo podem explicar essa situação, tendo em vista seus percentuais de inadequação: $89,9 \%$ e 89,6\%, respectivamente. Os resultados negativos para a existência de equipamentos públicos de SAN $(88,6 \%)$ e percentual de famílias chefiadas por mulheres $(84,8 \%)$ também contribuíram para esse resultado. Outros dois indicadores desta dimensão, número de moradores por domicílios e taxa de analfabetismo, igualmente apresentaram resultados desfavoráveis em mais de $50 \%$ dos municípios. 
A renda per capita média e a razão de renda foram classificadas como adequadas na maioria dos municípios. A cobertura do Programa Bolsa família (PBF) se mostrou apropriada em 46,9\% dos municípios, e aceitável em outros 43,5\%. Esses resultados contribuíram para que essa dimensão tivesse um maior percentual de municípios em situação de IA leve e moderada em detrimento da IA grave.

A tabela 4 mostra a associação existente entre a situação de IA nos municípios baianos, tendo em vista o resultado de todas as dimensões e o estado de IA na dimensão de AA. Dos municípios que foram classificados com IA moderada e grave na dimensão de AA, 75,9\% (268/353) estavam na mesma situação na avaliação geral de SAN; entre os que se enquadraram em IA leve na dimensão de AA, 57,6\% (34/59), encontravam-se em IA leve na avaliação englobando todas as dimensões. Portanto, houve uma associação estatisticamente significante $(\mathrm{p}<0,000)$ entre estar em IA nas formas mais graves na dimensão de AA e a IA moderada e grave global nos municípios.

Tabela 4. Associação entre a situação de IA de municípios do Estado da Bahia englobando todas as dimensões e a dimensão de Acesso aos Alimentos. Salvador - BA, 2015.

\begin{tabular}{|c|c|c|c|c|c|}
\hline \multirow{2}{*}{\multicolumn{2}{|c|}{$\begin{array}{c}\text { Situação de IA na dimensão } \\
\text { de Acesso aos Alimentos }\end{array}$}} & \multicolumn{3}{|c|}{ Situação de IA englobando todas as dimensões } & \multirow[b]{2}{*}{$\mathrm{p}$ valor } \\
\hline & & $\begin{array}{c}\text { IA moderada e } \\
\text { grave } \\
\text { n }(\%)\end{array}$ & $\begin{array}{l}\text { IA leve } \\
\text { n }(\%)\end{array}$ & $\begin{array}{l}\text { Total } \\
\mathrm{n}(\%)\end{array}$ & \\
\hline \multirow{2}{*}{$\begin{array}{l}\text { Acesso aos } \\
\text { Alimentos }\end{array}$} & $\begin{array}{l}\text { IA moderada } \\
\text { e grave }\end{array}$ & $268(75,9)$ & $85(24,1)$ & $353(100)$ & \multirow{2}{*}{0,000} \\
\hline & IA leve & $25(42,4)$ & $34(57,6)$ & $59(100)$ & \\
\hline \multicolumn{2}{|c|}{ Total } & $293(71,1)$ & $119(28,9)$ & $412(100)$ & \\
\hline
\end{tabular}

Neste estudo, constatou-se associação entre a situação de IA na dimensão de Acesso aos Alimentos e o Índice de Desenvolvimento Humano Municipal (IDHM) de municípios do Estado da Bahia (Tabela 5). Dos municípios que foram classificados com IA moderada e grave na dimensão de AA, 60,1\% apresentaram IDHM muito baixo e baixo e 24,6\%, IDHM médio. Assim, houve uma associação estatisticamente significante $(\mathrm{p}<0,000)$ entre estar em IA moderada e grave e ter o IDHM muito baixo e baixo. 
Tabela 5. Associação entre a situação de IA na dimensão de Acesso aos Alimentos e o Índice de Desenvolvimento Humano de municípios do Estado da Bahia. Salvador - BA, 2015.

\begin{tabular}{|c|c|c|c|c|c|}
\hline \multirow{2}{*}{\multicolumn{2}{|c|}{$\begin{array}{c}\text { Situação de IA na dimensão de } \\
\text { Acesso aos Alimentos }\end{array}$}} & \multicolumn{3}{|c|}{ Acesso aos Alimentos } & \multirow[b]{2}{*}{$\mathrm{p}$ valor } \\
\hline & & $\begin{array}{c}\text { AI moderada e } \\
\text { grave } \\
\mathrm{n}(\%)\end{array}$ & $\begin{array}{l}\text { IA leve } \\
\mathrm{n}(\%)\end{array}$ & $\begin{array}{l}\text { Total } \\
\mathrm{n}(\%)\end{array}$ & \\
\hline \multirow{3}{*}{$\begin{array}{c}\text { Índice de } \\
\text { Desenvolvimento } \\
\text { Humano } \\
\text { Municipal }\end{array}$} & $\begin{array}{l}\text { Muito baixo } \\
\text { e Baixo }\end{array}$ & $247(60,1)$ & $12(2,9)$ & $259(63,0)$ & \multirow{3}{*}{0,000} \\
\hline & Médio & $101(24,6)$ & $41(10,0)$ & $142(34,6)$ & \\
\hline & $\begin{array}{l}\text { Alto e Muito } \\
\text { alto }\end{array}$ & $3(0,7)$ & $7(1,7)$ & $10(2,4)$ & \\
\hline \multicolumn{2}{|c|}{ Total } & $351(85,4)$ & $60(14,6)$ & $411(100)$ & \\
\hline
\end{tabular}

\section{Discussão}

As pesquisas de avaliação da SAN domiciliar no Brasil usam a ferramenta da EBIA, o que tem contribuído para melhor conhecimento da situação e de seus determinantes. ${ }^{7}$ Sua aplicação, no entanto, não contempla todas as dimensões da avaliação da SAN, focando principalmente as variáveis da dimensão de AA, e os dados produzidos não alcançam o nível municipal. Dessa forma, a aplicação do protocolo de indicadores é mais uma opção de ferramenta para avaliar um fenômeno tão complexo quanto a SAN, complementando a metodologia da EBIA.

No Brasil, segundo dados da última PNAD realizada em 2013, que utiliza a ferramenta da EBIA, ocorreu melhora na situação de domicílios particulares em SAN para 77,4\%, sendo registrado anteriormente $65,1 \%$ e 69,8\%, em 2004 e 2009, respectivamente. ${ }^{7}$ Porém, a IA, em algum grau, ainda atinge cerca de 14,7 milhões de domicílios brasileiros (22,6\%), e a situação mais preocupante é observada nas regiões Norte e Nordeste do país (36,1\% e 38,1\%, respectivamente). Na Bahia, a prevalência de IA atinge cerca de $38 \%$ dos domicílios, ou seja, este problema ainda afeta uma grande parcela da população brasileira e baiana. ${ }^{7}$

O protocolo da EBIA foi utilizado para realização de estudos em municípios do Nordeste, que demonstraram elevadas prevalências de insegurança alimentar. ${ }^{14-16}$ Bittencourt et al. encontraram em seu estudo, no município de Salvador, em 2007, IA em 71,3\% dos domicílios particulares de famílias de escolares da rede pública. ${ }^{14}$ 
Estudos realizados em outros países como Nigéria e Índia, com perfil socioeconômico similar ao do Brasil, vêm demonstrando elevada prevalência de IA. ${ }^{17,18}$ Segundo a FAO, 805 milhões de pessoas no mundo estariam em situação de fome crônica, o que caracteriza a forma mais grave da insegurança alimentar. ${ }^{9}$

Neste estudo, evidenciou-se, na análise geral, que a maioria dos munícipios baianos estão em IA e somente dois estão em situação de SAN. A maior prevalência encontrada foi de IA moderada, ou seja, uma situação de maior vulnerabilidade à privação de alimentos e à fome. Resultados semelhantes foram encontrados no estudo de Pereira, no qual, mediante a aplicação do protocolo de indicadores em 57 municípios baianos de diferentes portes populacionais, constatou que 100\% dos municípios apresentavam IA, desde condições mais leves até situações mais graves, com maior prevalência de IA moderada. ${ }^{1}$

Na avaliação da SAN por dimensões, observa-se que as de AA e DA apresentaram uma alta prevalência de municípios baianos em IA moderada e grave. Na dimensão de AA, a maioria dos municípios estão com IA moderada. Nesta foram avaliados aspectos como renda, emprego, escolaridade, densidade domiciliar, famílias de baixa renda atendidas pelo PBF, famílias chefiadas por mulheres e a existência de equipamentos públicos de SAN, no período de 2010-2014.

Ao avaliar se a dimensão de AA é determinante para o quadro de IA nos municípios do Estado da Bahia, encontrou-se associação estatisticamente significante. Chatterjee observou resultados semelhantes em estudo que revelou que a insegurança alimentar grave foi significativamente associada com menor renda familiar mensal e outras medidas de status socioeconômicos. ${ }^{17}$ A dimensão de AA é muito complexa e se relaciona com aspectos socioeconômicos e físicos que interferem na aquisição de alimentos pela população/família, englobando assim a disponibilidade de recursos para aquisição de alimentos e todas as necessidades básicas para a garantia de SAN, além dos fatores que afetam os recursos disponíveis. ${ }^{9,19}$

Houve, neste estudo, associação entre a situação de IA na dimensão de Acesso aos Alimentos e o IDHM de municípios do Estado da Bahia. A pobreza demonstrada nos municípios baianos através do IDHM pode ser atribuída principalmente às variáveis renda, educação e saúde. Segundo Janvry \& Sadoulet, a pobreza é multidimensional, estando associada não apenas arenda, mas a outros diversos elementos, em especial a inadequadas condições de educação e saúde. ${ }^{20}$ Hoffmann afirma em estudo que a educação é uma das principais formas para ter ascensão social e de reduzir a desigualdade da distribuição da renda no Brasil. ${ }^{21}$

Na economia mercantil brasileira, o acesso permanente aos alimentos é determinado pelo poder aquisitivo das pessoas, ou seja, dispor de renda para comprar os alimentos. Sendo assim, a fome no Brasil está associada à pobreza em grande parte da população, que tem rendimentos 
muito baixos. ${ }^{21} \mathrm{~A}$ IA emerge desse contexto e está diretamente associada à fome nas populações, sendo a pobreza uma das causas estruturais da IA nos municípios baianos. ${ }^{22}$

Entre os municípios baianos, os indicadores de proporção de pessoas com baixa renda e percentual da população com 15 anos ou mais e menos de 4 anos de estudo tiveram percentuais de inadequação elevados, seguidos dos indicadores existência de equipamentos públicos de SAN e percentual de famílias chefiadas por mulheres. No estudo de Chatterjee, as famílias chefiadas por mulheres, cuja maior parcela da renda advém de pessoas menos escolarizadas, estavam mais propensas à insegurança alimentar grave. ${ }^{17}$

A escolaridade da população influencia na escolha e preparo dos alimentos, e principalmente na capacitação para alcançar uma melhor remuneração no trabalho, que, por sua vez, possibilitaria maior acesso aos alimentos. ${ }^{4}$ Estudos vêm demonstrando que a renda é diretamente proporcional à escolaridade. ${ }^{23-25}$ Segundo Tannen, o aumento salarial para cada ano adicional de estudo foi de $12,8 \%$ para o primeiro ciclo e $8,1 \%$ para o segundo, do ensino básico; para o segundo grau e ensino superior, foi de $15,7 \%$ e $23,4 \%$, respectivamente. Mas um ano adicional do ensino superior apresenta maior aumento sobre os salários. ${ }^{24}$

Salvato et al. afirmam que a desigualdade de renda e a de escolaridade são fortemente correlacionadas e que a escolaridade média nas regiões mais pobres é cerca de três anos menor que nas regiões mais ricas. ${ }^{23}$ Esse estudo corrobora os resultados encontrados neste trabalho para os indicadores proporção de pessoas com baixa renda e percentual da população com 15 anos ou mais e menos de 4 anos de estudo nos municípios baianos.

Outros indicadores pertencentes à dimensão de AA, a exemplo da densidade de moradores por domicílio, implicam maior diluição da renda, reduzindo, assim, o recurso per capita para aquisição e utilização de bens e serviços essenciais à manutenção do estado de saúde, principalmente à aquisição de alimentos. Este fator associado à ocorrência de concentração de renda no município demonstra uma desigualdade, em que a minoria da população apresenta capital acumulado, enquanto a maioria das pessoas possuem baixa renda, sendo, dessa maneira, insuficiente para atender às necessidades básicas de consumo, bem como à aquisição de alimentos em quantidade e qualidade satisfatórias. Essas situações estão relacionadas diretamente com a IA. ${ }^{4}$

Ações voltadas para o aumento da disponibilidade de renda das populações mais carentes, através de políticas de redução da pobreza e das desigualdades sociais, políticas de educação e qualificação profissional e programas de acesso ao crédito se fazem necessárias para aumento da SAN, especialmente em relação às carências agudas. ${ }^{8}$ 


\section{Conclusão}

Os resultados do presente estudo mostraram alta prevalência de IA moderada e leve nos municípios baianos. A IA global nesses municípios mostrou haver associação com a ocorrência de IA na dimensão de AA. Os indicadores da dimensão de AA que mais contribuíram para esse quadro foram proporção de pessoas com baixa renda e percentual da população com 15 anos ou mais e menos de 4 anos de estudo.

A pobreza e a dificuldade ao acesso regular e permanente a alimentos são influenciadas por fatores como renda, educação e saúde, mensurados no IDHM. O IDHM muito baixo/baixo dos municípios baianos está associado à IA nas formas mais graves existente na dimensão de Acesso aos Alimentos. Apesar da dimensão de Acesso aos Alimentos ter forte relação com insegurança alimentar, não é suficiente para explicá-la. O embate da IA perpassa diferentes áreas do governo e engloba as quatro dimensões de SAN: DA, CA, AA e UBN.

O protocolo de indicadores utilizado neste trabalho pode ser empregado nos instrumentos de coleta de informações de vários tipos de pesquisas, cujos objetivos sejam estudar este fenômeno tão abrangente que é a SAN. Também pode levar à compreensão das causas da IA, compondo-se como um quadro capaz de auxiliar o desenvolvimento de ações e políticas de enfrentamento da IA em nível municipal de forma mais efetiva. Além de se comportar como um instrumento de baixo custo, fácil aplicação e sensível aos diferentes estados da SAN por contemplar as quatro dimensões.

\section{Referências}

1. Pereira MHQ. Avaliação da Segurança Alimentar e Nutricional: contribuições em âmbito municipal [dissertação]. [Salvador]: Universidade Federal da Bahia; 2014.

2. Santos SMC, Santos LMP. Avaliação de políticas públicas de segurança alimentar e combate à fome no período de 1995-2002: 1 - Abordagem metodológica. Cad Saúde Pública 2007; 23(5):1029-1040.

3. Brasil. Decreto-Lei 11.346, de 15 de setembro de 2006. Cria o Sistema Nacional de Segurança Alimentar e Nutricional SISAN, com vistas em assegurar o Direito Humano à Alimentação Adequada e dá outras providências. Diário Oficial da União 18 set. 2006.

4. Burlandy L. A construção da política de segurança alimentar e nutricional no Brasil: estratégias e desafios para a promoção da intersetorialidade no âmbito federal de governo. Ciênc Saúde Colet. 2009; 14(3):851-860.

5. Panelli-Martins BE, Santos SMC, Assis AMO. Segurança alimentar e nutricional: desenvolvimento de indicadores e experimentação em um município da Bahia, Brasil. Rev. Nutr. 2008; 21(Supl.):65s-81s.

6. Takagi M. A Implantação da Política de Segurança Alimentar e Nutricional no Brasil: seus limites e desafios [tese]. [Campinas, SP]: Instituto de Economia, Unicamp; 2006. 
7. Instituto Brasileiro de Geografia e Estatística. Pesquisa Nacional por Amostra de Domicilio (PNAD) - Segurança Alimentar 2013. Rio de Janeiro: IBGE; 2014.

8. Brasil. Conselho Nacional de Segurança Alimentar e Nutricional. A Segurança Alimentar e Nutricional e o Direito Humano à Alimentação Adequada no Brasil Realização - Indicadores e Monitoramento - da Constituição de 1988 aos dias atuais. Brasília: CONSEA; 2010. 284 p.

9. Organização das Nações Unidas para a Alimentação e a Agricultura. O Estado da Insegurança Alimentar no Mundo, 2014. Fortalecimento de um ambiente favorável para a segurança alimentar e nutrição. Roma: FAO; 2014. 8 p. [acesso em: 15 nov. 2015]. Disponível em: http://www.fao.org/3/ai4037o.pdf

10. Cotta RMM, Machado JC. Programa Bolsa Família e segurança alimentar e nutricional no Brasil: revisão crítica da literatura. Rev Panam Salud Publica 2013; 33(1):54-60.

11. Panelli-Martins BE. Analise de método de avaliação da segurança alimentar e nutricional: uma contribuição à política municipal de SAN [dissertação]. [Salvador]: Universidade Federal da Bahia; 2007.

12. Jannuzzi PM. Indicadores para diagnóstico, monitoramento e avaliação de programas sociais no Brasil. RSP 2005; 56(2):137-160.

13. Programa das Nações Unidas para o Desenvolvimento. Índice de Desenvolvimento Humano Municipal Brasileiro. Brasília: PNUD; 2013. 95 p. Atlas do Desenvolvimento Humano no Brasil 2013.

14. Souza Bittencourt L, Chaves dos Santos SM, Jesus Pinto E, Aliaga MA, Cássia Ribeiro-Silva R. Factors associated with food insecurity of households of public schools students in the City of Salvador, Bahia. J Health Popul Nutr. 2013; 31(4):471-9.

15. Vianna RPT, Hromi-Fiedler AJ, Segall-Correa AM, Pérez-Escamilla R. Household food insecurity in small municipalities in Northeastern Brazil: a validation study. Food Security 2012; 4(2):295-303.

16. Ribeiro JCS. Avaliação da insegurança alimentar e nutricional em um consórcio de segurança alimentar e desenvolvimento local - CONSAD na Bahia. 2012 [dissertação]. [Salvador]: Universidade Federal da Bahia, Escola de Nutrição, 2012.

17. Chatterjee N. Food insecurity in urban poor households in Mumbai, India. Food Security 2012; 4(4):619-632.

18. Akerele D. Food insecurity and coping strategies in South-West Nigeria. Food Security 2013; 5(3):407-414.

19. Timmer PC. The macro dimensions of food security: economic growth, equitable distribution, and food price stability. Food Policy 2000; 25(3):283-295.

20. Janvry A, Sadoulet E. La inversión en desarollo rural es buen negócio. In: Echevererria R, editor. Desarollo de las economias rurales en América Latina y el Caribie. Washiington, DC: BID; 2001. 41 p.

21. Hoffmann R. Determinantes da Insegurança Alimentar no Brasil: Análise dos Dados da PNAD de 2004. Segur. Aliment. Nutr. 2008, 15(1): 49-61. 
22. Maluf RS. Ações públicas locais de abastecimento alimentar. São Paulo, Polis Papers: Polis Assessoria, Formação de Estudos em Políticas Sociais 1999; 99(5):1-42.

23. Salvato MA, Ferreira PCG, Duarte AJMA. O impacto da escolaridade sobre a distribuição de renda. Estud. Econ. 2010; 40(4):753-791.

24. Tannen M. New estimates of the returns to schooling in Brazil. Econ. Educ. Rev. 1991; 10(2):123-135.

25. Teixeira WM, Menezes-Filho NA. Estimando o retorno à educação do Brasil considerando a legislação educacional brasileira como um instrumento. Revista de Economia Política. 2012; 32(3):479-496.

Recebido: $13 / 6 / 2016$

Revisado: 25/10/2016

Aceito: 27/10/2016 